\title{
PATH-DEPENDENCY, INSTITUCIONES POLÍTICAS Y REFORMAS ELECTORALES EN PERSPECTIVA COMPARADA*
}

\author{
ANDRÉ MARenco \\ Universidad Federal de Río Grande del Sur, Brasil
}

\begin{abstract}
Resumen
Este trabajo examina en qué medida la adopción de cambios en las reglas electorales son gradualmente condicionados por la configuración previa electoral. Las reformas electorales, una vez aprobadas, tienden a adquirir una intención incremental y son influenciadas por las reglas previas. Esto implica que las reformas electorales siguen un modelo path-dependent. Las reformas electorales son menos probables cuanto mayor es el tiempo de funcionamiento del modelo electoral previo y, si son adoptadas, presentarán márgenes de innovación limitados por el formato institucional anterior. Para analizar estas hipótesis, estudio 84 reformas electorales promovidas al interior de 40 países con sistemas políticos competitivos entre 1800 y 2002. Dos aspectos de los sistemas electorales son especialmente escrutados: las transformaciones en los modelos de representación electoral (mayoritarios, representación proporcional, mixtos) y los tipos de ordenamiento de los candidatos (sea por listas cerradas o voto preferencial). Los resultados apoyan las premisas que orientaron el estudio, mostrando los efectos restrictivos ejercidos por el tiempo y por las instituciones anteriores sobre los cambios electorales.
\end{abstract}

Abstract

This work examines to what extent changes in electoral institutions are conditioned by the previous electoral configuration. Electoral reforms, once approved, tend to acquire incremental intention and, consequently, are influenced by the previous rules, implying that they follow a path-dependent model. Electoral reforms are less probable the longer the time of existence of the previous electoral system and, if adopted, they show restricted margins of innovation. In order to analyze these hypotheses, I study 84 electoral reforms promoted in 40 countries with competitive political systems between 1800 and 2002. Two aspects are considered within the arena of electoral reform: changes in the modes of representation (plurality-majority, proportional representation, and mixed) and the types of ordering of legislative candidates (closed lists or preferential vote). The results support the premises of this study showing the restrictive effects exerted by time and the previous institutions on electoral changes.

PALABRAS CLAVE •Instituciones políticas • Reformas electorales $\bullet$ Path-dependency • Fórmulas electorales

\section{INTRODUCCIÓN}

¿Bajo qué circunstancias las poliarquías promueven reformas en las reglas que regulan la competencia electoral? ¿Cómo podríamos explicar la opción por diferentes modelos de conversión de votos en escaños legislativos, si las fórmulas electorales, una vez adoptadas y puestas en marcha, ejercen efectos importantes sobre estrategias de partidos y electores? ¿Qué factores

* Este artículo fue traducido al español por Iván Bambarén. 
condicionan la decisión por determinada reforma electoral que condicionará, posteriormente, la distribución de preferencias del electorado?

Este trabajo busca examinar en qué medida la adopción de cambios en las instituciones y reglas electorales son gradualmente condicionados por la configuración previa del statu quo institucional e influenciados por las reglas en vigor. Esto implica que las reformas electorales seguirían un modelo path-dependent: las reformas electorales serían menos probables cuanto mayor el tiempo de funcionamiento del modelo electoral previo y, una vez adoptadas, presentarían márgenes de innovación coactadas por el formato institucional previo. Para analizar este problema, inicialmente fueron examinadas 203 reformas electorales. Sin embargo, se optó por concentrar el foco del análisis en aquellas reformas promovidas al interior de sistemas políticos competitivos, reduciendo el número de casos a 101 (47,7\%). Por fin, fueron consideradas solamente las reformas electorales en estricto senso, o sea, cuando una institución electoral cambia a otra institución electoral en funcionamiento, en un contexto competitivo, reduciendo el examen a 84 casos. Dos aspectos de los sistemas electorales son especialmente escrutados: transformaciones en los modelos de representación electoral (mayoritarios, representación proporcional, mixtos) y el tipos de ordenamiento de los candidatos (listas cerradas y voto preferencial). Ver Anexo al final del trabajo, donde se enumeran y describen todas las reformas estudiadas en profundidad.

La primera sección del trabajo parte de la constatación de que, si por un lado, la investigación contemporánea sobre instituciones políticas ofrece respuestas consistentes sobre los propósitos producidos por instituciones sobre estrategias, acciones y resultados políticos, por otro, no ha sido capaz de promover avances igualmente consistentes en la explicación sobre la formación, variación, estabilidad y cambio institucional. En otras palabras, hoy sabemos que las instituciones explican procesos y resultados políticos, pero sabemos muy poco sobre cómo son formadas las instituciones.

La segunda parte del trabajo analiza la evidencia partiendo de un examen de las reformas electorales, según el modelo adoptado y el período en que son implementadas. A continuación, se realiza un inventario de esas reformas electorales conforme su flujo, identificando el modelo electoral previo y la alternativa institucional adoptada. Finalmente, se busca medir el impacto ejercido por el tiempo, evaluando la medida en que la longevidad temporal de una fórmula electoral reduce las probabilidades de su sustitución, y los factores que influyen en las reformas, según intervalos temporales distintos de duración del statu quo. De la misma forma, se analizó la resistencia institucional de los diferentes modelos focalizados en el análisis. Los resultados apoyan las premisas que orientaron el estudio, mostrando los efectos restrictivos ejercidos por el tiempo y por las instituciones previas sobre los cambios electorales.

\section{II. ¿CÓMO SON ESCOGIDAS LAS INSTITUCIONES?}

La agenda de investigación de la ciencia política convergió, en las últimas dos décadas, hacia la premisa que las instituciones "importan", siendo estas eficaces en la reducción de los costos de transacción sociales y resolviendo problemas de coordinación colectiva a través de la inducción de preferencias, comportamientos y outcomes públicos (North, 1990; Ostrom, 1990; Shepsle, 1995). El nuevo institucionalismo se ha centrado en los procesos por los cuales las instituciones, 
concebidas como set de constricciones y oportunidades contextuales, moldean el comportamiento político estructurando resultados probables para interacciones entre agentes sociales (Hall y Taylor, 1996; Peters, 1999; Diermeier y Krehbiel, 2003). Más allá de una valorización de la configuración institucional como variable independiente y del énfasis atribuido al condicionamiento ejercido por reglas y estructuras sobre acciones individuales, lo que parece implícito en el modelo es la dirección de la causalidad presente en la relación instituciones/agentes: las instituciones condicionan las acciones y las estrategias de los agentes y son resistentes a éstas (Diermeier y Krehbiel, 2003: 130). Por otro lado, su definición de estructuras políticas como colección de reglas de comportamiento, normas y roles relativamente invariables en relación a la circulación de individuos y sus idiosincrasias (March y Olsen, 1983: 741), proyecta una cuestión relativa a las condiciones precisas en que instituciones son escogidas, adquieren estabilidad y, por supuesto, rutinas. $\mathrm{Si}$, por un lado, la introducción de instituciones en los modelos explicativos para acción colectiva y opciones individuales permitió resolver la paradoja de la cooperación, por otro, no parece posible desconocer la presencia de lagunas relacionadas a la interpretación sobre la formación y origen de esas instituciones, las condiciones que contribuyen para su manutención y estabilidad y los factores que provocan su decadencia y el cambio institucional. Esas cuestiones, probablemente, constituyan el talón de Aquiles de la escuela neoinstitucionalista, para las cuales las respuestas parecen todavía insuficientes (Peters, 1997; Pierson, 2004).

Si las instituciones importan, como afirma la consigna neoinstitucionalista, ellas no son un dato de la naturaleza, no emergen por generación espontánea, ni se mantienen por el cumplimiento de necesidades o funciones sociales. Diferente de un dios ex machina -artefacto introducido súbitamente para solucionar un problema formal presente en la reconstitución analítica-, es preciso explicar las opciones y las condiciones que generan el surgimiento de tipos específicos de instituciones, las causas de la variedad de modelos institucionales, sus factores de continuidad y las rupturas institucionales. El desafío puesto para la agenda de las teorías institucionales consiste en hacer que las respuestas sean endógenas a las interpretaciones sobre instituciones políticas, ofreciendo elementos capaces de explicar su existencia, así como incorporar la diacronía como dimensión interpretativa de su dinámica.

Pero, ¿cómo surgen las instituciones? La distinción propuesta por Diermeier y Krehbiel (2003) entre institucionalismo y teoría de instituciones intenta ofrecer una solución al desafío de hacer endógena la explicación para la formación institucional. En cuanto la primera deduce comportamientos y outcomes a partir de las coacciones fijadas por una determinada configuración institucional, el énfasis de la teoría de instituciones sería explicar la formación, variación, estabilidad y cambio en la configuración de las instituciones políticas (Diermeier y Krehbiel, 2003: 130). Adoptando el modelo de equilibrio de Nash, Diermeier y Krehbiel sugieren que la opción institucional por los agentes políticos racionales estaría condicionada por instituciones de segundo orden. En otras palabras, las instituciones que condicionan comportamientos y resultados son producto de cálculo y opciones efectuadas por los agentes, pero esa opción es coactada por la presencia de instituciones previas, en una explicación que en el límite lleva a un ejercicio de infinitas regresiones, como en el caso de las matrioshkas rusas.

Las restricciones en los márgenes de cambio institucionales son mayores en circunstancias de equilibrio, resultante de la percepción de riesgos de empeoramiento en la posición relativa con el cambio, costos de transacción elevados para el convencimiento de los demás actores, o de la 
existencia de mecanismos de self-enforcement, que tornan la alteración del statu quo un proceso difícil y de ocurrencia poco probable. Por ejemplo, reglas que establecen quórum elevado para el cambio de dispositivos constitucionales pueden constituir, así, un procedimiento que contribuye a la inercia institucional. En dirección semejante, el número y posición de los veto-players reducen el objetivo de probabilidades para cambio en el statu quo e inestabilidad institucional (Tsebelis, 2002). El punto aquí consiste en que el análisis no puede dejar de lado un esfuerzo en disecar la constitución y preferencias de los agentes políticos: condiciones, como en juegos interactivos, que tornan más probable el conocimiento y previsión del comportamiento de los demás, alterando la estructura de premios y sanciones para el cambio o continuidad institucional, dependen de las condiciones de ingreso y movilidad en el interior de elites políticas, reduciendo costos de transacción, ampliando la información disponible y favoreciendo la oportunidad para compromisos y cumplimiento de acuerdos, o inversamente, tornando más inciertas e imprevisibles las acciones de cada miembro, ante los ojos de los demás. De la misma forma, las curvas de indiferencia de los veto players no son fijadas exógenamente al proceso político, pudiendo ser alteradas con base en la información y la utilidad representada por la propia institución para grupos específicos de actores.

La respuesta alternativa al problema de la explicación para la opción, formación y estabilidad institucional, está relacionada a los costos elevados para la reversión de políticas o reglas institucionales, presentes bajo la forma de path-dependency. Las condiciones originales presentes en la formación institucional y en la secuencia cronológica de los eventos -indicando que en este caso el orden temporal de los factores altera el producto final- son responsables por la generación de una inercia estructural. El tiempo aquí presenta efectos de retornos crecientes impidiendo con su prolongamiento el margen de cambio institucional (North, 1990: 95; Pierson, 2004: 18).

Por otro lado, la sugerencia de Pierson (2004) que la contingencia preside la formación institucional puede representar una pista para comprender el origen de estructuras institucionales no como respuesta a designios funcionales, sino como producto de eventos discretos y de consecuencias no previstas de estrategias volcadas hacia objetivos inmediatos y circunstanciales. La indicación de Pierson que procesos de tipo path-dependent involucran una combinación de efectos de larga duración (por los incluidos elevados costos de reversión), con factores causales fijados en cortos recortes temporales, parece constituir una buena pista, capaz de dispensar el determinismo y la irreversibilidad histórica.

\section{DE LAS CONSECUENCIAS POLÍTICAS DE LAS INSTITUCIONES ELECTORALES A LAS CAUSAS PARA LA OPCIÓN DE LAS REGLAS DE VOTACIÓN}

Existe una importante literatura sobre las consecuencias políticas de las leyes electorales: los incentivos al voto estratégico producido por diferentes fórmulas de conversión de votos en bancas legislativas (Duverger, 1954; Lijphart, 1990; Cox, 1997; Rose, 2000; Norris, 2004; Blais, Young y Turcotte, 2005), el impacto de los mecanismos de remanentes y de la magnitud de los distritos (Rae, 1967; Taagepera, 1984; Taagepera y Shugart, 1989) o, incluso, los efectos de inducción sobre reputaciones personales o partidarias, provocados por diferentes modelos de ordenamiento de listas intrapartidarias (Cain, Ferejohn y Fiorina, 1987; Carey y Shugart, 1996; 
Crisp et al., 2004). Todos estos ejemplos parecen constituir casos persuasivos de la premisa que las instituciones "importan" para explicar decisiones, estrategias y comportamientos políticos. Sin embargo, habiendo conocido sucesivos ablandamientos posteriores, las "leyes" se convirtieron en "tendencias", más tarde en "frenos" y "aceleradores", y por fin, en "presiones"; la proposición original de Duverger sugería que sistemas de pluralidad deberían conducir a un dualismo con alternancia entre los dos partidos mayores, sistemas de mayoría con segunda vuelta generarían un multipartidarismo flexible, interdependiente y relativamente estable, y la representación proporcional, un sistema multipartidario, más rígido, con partidos independientes y estables. El impacto más significativo estaría relacionado al número de partidos, destacando las consecuencias asociadas a los sistemas uninominales de mayoría simple bajo la forma de la reducción del universo partidario a dos competidores significativos. Para esto, concurren factores “mecánicos", por la subrepresentación de la tercera fuerza, y "psicológicos", en la tendencia del elector para concentrar su voto en los dos mayores partidos. Al mismo tiempo, las diferencias en las personalidades e interacciones entre las organizaciones políticas en sistemas multipartidarios serían provocadas por la adopción de segundas vueltas -que ofrecen la oportunidad para la expresión de toda la diversidad ideológica en el primer turno, al mismo tiempo que inducen un modelo de convergencia bipolar en segunda instancia (Fisichella, 1984)- 0, por la representación proporcional, incentivando la afirmación de identidades diferenciadas.

Una versión mitigada de la proposición original de Duverger ha sido explotada en los trabajos más recientes de Sartori (1996). Con el objetivo de recuperar la gran parte de los efectos de la ingeniería electoral sobre el formato de los sistemas partidarios, agotado por las críticas en las últimas décadas, Sartori ofrece, prudentemente, un modelo más modesto limitando su esfuerzo predictivo a los sistemas electorales "fuertes" comprendidos como aquellos que generan efectos sobre el número de partidos. Ahora, no todas las fórmulas electorales producen consecuencias quedando esta aptitud restricta a las reglas de mayoría simple. En este caso, según Sartori, las reglas pueden contribuir para congelar un bipartidarismo preexistente, o incluso, ejercer un "efecto reductor" sobre el número de partidos, cuando se dé su implantación. Con todo, esta comprensión solamente ocurrirá en la presencia de: [1] un sistema partidario estructurado, lo que debería explicar la excepción representada por la India; y [2] una "dispersión cruzada de los electorados", ausente en Canadá, justificando su diferencia a la predicción del modelo. El problema es que hecha la concentración de foco (pluralidad), y establecidas las excepciones, restarán cuatro casos explicables por el paradigma: Inglaterra, Estados Unidos, Australia y Nueva Zelandia, que han tenido en común, además de la cultura anglosajona, un sistema electoral uninominal de mayoría simple.

Las fórmulas para la conversión de votos en escaños legislativos corresponden a una parte de la ingeniería institucional dedicada a la producción de la representación política. En el caso de las instituciones de representación proporcional, se torna relevante fijar el procedimiento de distribución de los asientos partidarios entre sus candidatos parlamentarios. Esto se da tanto por listas cerradas escogidas dentro de las organizaciones partidarias o por el voto preferencial expresado por el elector.

Ha sido muy atractivo, para la literatura, fijar una conexión entre: [a] reglas electorales basadas en voto preferencial, [b] incentivos para la promoción de reputaciones personalizadas como estrategia dominante y [c] un modelo de conexión electoral basado en el refuerzo de lealtades 
parroquiales y en el uso del pork barrel en el intento de reducir la incertidumbre generada por elevados costos de información al elector, provocados por el incremento en el número de candidatos individuales y por la competencia intrapartidaria (Carey y Shugart, 1996: 419; Norris, 2002: 4-5; Crisp et al., 2004: 831).

La matriz para los diagnósticos producidos sobre los efectos de diferentes reglas de ranking electoral de candidatos partidarios puede ser localizada en el modelo de electoral connection, originalmente formulado por David Mayhew (1974). Investigando las estrategias de carreras de legisladores norteamericanos, Mayhew encontró evidencias de que la búsqueda de reelección los induciría a cultivar reputaciones personalizadas, y a promover una oferta de incentivos selectivos y muy particulares para su electorado como mejor respuesta para esta estructura de oportunidades. La relación entre sustento de la carrera política, búsqueda de votos personalizados y mandatos legislativos orientados para la captura de recursos dirigidos para el reducto electoral-focalizado en distritos uninominales- fue identificada entre otros contextos, además de aquel observado originalmente (Cain, Ferejohn y Fiorina, 1987).

Uno de los primeros trabajos a emplear en el modelo de conexión electoral, para interpretar la dinámica de la competencia bajo el voto preferencial, fue presentado por Scott Mainwaring (1991). Examinando las reglas electorales que operan en Brasil desde 1946, Mainwaring indicó que en el anteceder a la institucionalización de organizaciones partidarias nacionales, las listas abiertas habrían creado incentivos para la débil disciplina y fidelidad de los candidatos en relación a sus partidos. De esa forma, al incentivar la competencia intrapartidaria, el mecanismo de lista abierta sería el responsable por provocar infidelidad, migración interpartidos, menor disciplina legislativa, votos personalizados, reproduciendo una situación endémica de fragilidad partidaria.

A partir de diferentes modelos de listas electorales, Carey y Shugart (1996) elaboraron un cuadro comparativo para calcular los incentivos institucionales en la generación de reputación partidaria o personal como recurso para carreras políticas, ofreciendo una puntuación para medir los medios de control a disposición de los líderes del partido: prerrogativa de las nominaciones y ordenamiento de los electos, transferencia de votos, restricciones a la competencia intrapartidaria y la existencia de barreras a la formación de nuevos partidos, ocasionada por la magnitud de los distritos electorales. En esta dirección, lista abierta, candidatos natos y una elevada magnitud de las circunscripciones electorales incrementarían el potencial de competencia intrapartidaria, reduciendo, paralelamente, el control de liderazgo del partido sobre sus miembros y candidatos y ampliando el valor de reputaciones personales como capital político. En esta perspectiva, fórmulas mayoritarias uninominales, con nominación partidaria, como la del Reino Unido y Canadá, y representación proporcional (RP) con lista cerrada (Israel, España), serían ejemplos de instituciones que, al conferir mayor reserva de recursos a disposición de los líderes del partido, generarían menor incentivo a la mantención de reputaciones partidarias. En contraste, las múltiples listas partidarias con transferencia limitada de Colombia constituirían reglas con mayores incentivos para la captura de votos personales. En un caso como el brasileño, la transferencia interpartidaria de votos nominales podría ser un factor capaz de mitigar el efecto reputacional causado por el modelo de listas abiertas. Por otro lado, conforme a Carey y Shugart, el incremento de la magnitud electoral provocaría consecuencias opuestas, ampliando el valor de votos personalizados, como factor de distinción en la competencia intrapartidaria. 
Analizando seis casos nacionales en América Latina, Crisp et al. (2004) aislaron dos variables como recursos explicativos para la generación de modelos de conexión electoral: procedimientos partidarios de selección de candidaturas, de indicaciones centralizadas como en Chile, Costa Rica y Honduras, hasta descentralizadas, como en Colombia, pasando por los ejemplos intermedios de Argentina y Venezuela; paralelo, reglas electorales de lista cerrada o con competencia intrapartidaria (comprendiendo listas abiertas y listas subpartidarias). Así, las decisiones descentralizadas, sumadas a la competencia intrapartidaria, constituirían el contexto más favorable en la disputa por votos personalizados y políticas muy particularistas.

En síntesis, el argumento convencional producido por la literatura sugiere que bajo reglas de voto preferencial, en el cual el orden de los candidatos a ocupar los escaños generados por la cuota proporcional de cada partido es definida por la votación individual de cada candidato, la consecuencia será la adopción de estrategias basadas en la reputación personal, viabilizadas preferentemente por políticas distributivas de concesiones concentradas de recursos públicos: presupuesto, empleos y otras ventajas como condición para la mantención y/o movilidad en la carrera política.

El vacío existente, no obstante, reside en el poder explicativo para interpretar el origen de instituciones electorales que, bajo ciertas condiciones, provocan efectos previsibles sobre los agentes políticos. Para Boix (1999), la fuerza de partidos emergentes y la capacidad de los competidores tradicionales en resolver problemas de coordinación constituyen las explicaciones para el asunto del cambio en las instituciones electorales: cuando los partidos desafiantes son débiles y/o los partidos tradicionales se muestran capaces de resolver "problemas de coordinación" (Cox, 1997) la tendencia es a la mantención de la fórmula mayoritaria; inversamente, bajo la concurrencia de partidos desafiantes relevantes y delante la imposibilidad de coordinar votos del electorado conservador, la probabilidad sería la introducción de la representación proporcional. En dirección semejante Colomer (2003) prueba un modelo de equilibrio behavioral-institutional, sugiriendo que la dispersión y el pluralismo partidario previos provocan ajustes en las reglas electorales, generando incentivos para la sustitución de fórmulas mayoritarias por representación proporcional, indicando una dirección de causalidad inversa a la propuesta por Duverger (partidos condicionan un sistema electoral, y no lo contrario). En estos modelos, los cambios son producto de componentes exógenos, localizados en especial en la distribución de preferencias del electorado.

Este trabajo intenta dilucidar los efectos de tipo path-dependency producidos por las propias instituciones electorales, cuestionándose en qué medida la extensión temporal de las reglas electorales termina por inducir un efecto de positive feedback (Pierson, 2004), tornando en reversión y cambios poco probables, incluso después de alteraciones en el formato de la competencia en la arena electoral, provocadas por factores demográficos, políticos o culturales. 0 más aún, ¿condiciona la matriz institucional previa el objetivo de los cambios en la ingeniería electoral limitando el alcance de los cambios a variaciones graduales del modelo original?

\section{REFORMA ELECTORAL EN PERSPECTIVA}

A continuación el trabajo busca analizar procesos de cambio en las reglas electorales, en el ámbito nacional entre 1800 y 2004. Fueron consideradas como reformas electorales alteraciones en la 
fórmula de conversión de votos en escaños que involucraran la sustitución entre instituciones mayoritarias, mixtas y de representación proporcional. Fueron estudiados también aquellos cambios internos en instituciones mayoritarias (uninominal o plurinominal y plurality o runoff), así como variaciones entre los modelos de RP basados en el ordenamiento partidario previo de listas electorales (listas cerradas) o aquellas que adoptaron el procedimiento de voto preferencial para definir el orden y los ocupantes de la cuota de escaños proporcionales conquistadas por cada partido. Se buscó registrar el año en que ocurrió cada uno de los cambios electorales, la dirección de este cambio, factores contextuales, como régimen político, desempeño económico, las condiciones de competencia política, y la durabilidad de cada formato institucional, definido por el intervalo temporal entre una reforma y otra.

La distinción entre instituciones mayoritarias (MAY), mixtas (MIX) y de representación proporcional (RP) se presenta consolidada en la literatura y no ofrece mayores problemas (Lijphart y Grofman, 1984; Lijphart, 1999). Más compleja, sin embargo, parece la diferencia entre distintos procedimientos de ordenamiento de candidatos promovidos en sistemas de representación proporcional.

Por "listas cerradas" (LC) se entiende aquí el procedimiento en el cual los partidos presentan anticipadamente una relación ordenada de sus candidatos, restándole a los electores un sufragio impersonal en la lista de su preferencia. Las vacantes son distribuidas entre los candidatos partidarios conforme al orden previamente establecido, hasta completarse la cuota proporcional partidaria correspondiente. El ordenamiento partidario de listas puede ser encontrado, entre otros casos, en Argentina, Indonesia, Noruega, Portugal, España y África del Sur.

Las reglas del ranking de candidatos que permiten algún tipo de competencia intrapartidaria fueron clasificadas como de "voto preferencial" (VP). El modelo más usual de ranking definido por la interferencia del elector en la definición del orden final consiste en la "lista abierta", o no ordenada, encontrada en países como Brasil, Finlandia, Suecia, República Checa y Chile, y corresponde al formato en el cual los partidos indican sus candidatos sin un orden de preferencia previa, siendo prerrogativa de los electores definir esta jerarquía a través del voto nominal conferido al postulante de su opción. Sumados los votos de los candidatos de cada partido, establecida la cuota proporcional de escaños que le cabe a cada uno, las bancas son distribuidas conforme el orden de votos nominales obtenidos por cada candidato. Fueron consideradas también como voto preferencial, las "listas flexibles", como la del sistema electoral belga, cuando los partidos presentan listas electorales previamente ordenadas, existiendo, sin embargo, la posibilidad de que el elector altere este ordenamiento, en la medida que deposite votos preferenciales para algún candidato en cantidad suficiente para reposicionarlo en la lista final ordenada después del conteo de los sufragios. En el sistema de "lemas", como en el caso de Uruguay, el elector vota en una lista subpartidaria, determinando la cuota proporcional partidaria y, simultáneamente, la distribución intrapartidaria de los escaños, entre los diferentes lemas. Aunque el sublema constituya una lista preordenada, la definición de la nómina final de los candidatos partidarios electos depende de la distribución intrapartidaria de preferencias del electorado, permitiéndose ubicarse Uruguay entre los casos del voto preferencial (Colomer, 2004; Rose, 2000). Colombia presenta un mecanismo semejante. Sin embargo, su fórmula de conversión de votos en escaños basada en el cuociente electoral, combinado a la primacía de los mayores restos, elimina el recurso de la transferencia y el compartir intrapartidarios de los sufragios (Archer y Shugart, 2002). Por 
fin, el single transferible vote (voto único transferible), aplicado en Irlanda y Malta, permite una ordenación previa de las preferencias del electorado, dirigiendo la transferencia del voto, en caso que las primeras opciones sean desperdiciadas. Después de un primer conteo, se descartan las primeras opciones de los candidatos menos votados, así como los votos en exceso de aquellos que alcanzaron el cuociente electoral. Este procedimiento es repetido sucesivamente, hasta que los escaños en disputa sean completados.

Una duda que podría ocurrir a esta altura sería la de la posibilidad de clasificarse dentro de una categoría única de voto preferencial, procedimientos distintos como listas enteramente no ordenadas, listas previamente ordenadas, pero sujetas a alteración por los electores, "doble voto simultáneo", panachage, e incluso "voto único transferible". Sin desconocer las peculiaridades de cada uno de los procedimientos disponibles que prevén la interferencia del elector en la distribución de los escaños partidarios, su equivalencia parte de la premisa de que cualquier esfuerzo analítico de interpretación de procesos políticos implica en un ejercicio de reducción, por el cual algunas propiedades son ocultadas, en cuanto otras, salientes. Lo mismo pasa cuando se utiliza "representación proporcional" como categoría de análisis: a pesar de las diferencias existentes dentro de las instituciones que emplean RP, resultantes de diferentes fórmulas para la distribución de restos, magnitudes electorales, cuociente, tipos de listas y ocupación de los curules partidarios, la RP ha sido utilizada como una variable para explicar grados de proporcionalidad, dispersión partidaria o desempeño institucional (Grofman, 2005: 736). Lo que se está acentuando al emplearse el "voto preferencial" como una categoría analítica son sus semejanzas referente a la posibilidad de interferencia del elector y la competencia intrapartidaria, como condiciones para la definición de la ocupación de los escaños partidarios (Crisp et al., 2004: 830), procurando probar si los efectos, como consecuencia de este modelo, corresponden a aquellos previstos por la literatura.

En el intervalo de 204 años entre 1800 y 2004, fueron registrados 203 cambios de reglas electorales, involucrando 93 naciones. Francia (12), Grecia e Italia (9), España (8) y Portugal (7) presentaron las mayores reincidencias de reformas, sugiriendo la inestabilidad de las instituciones electorales adoptadas en estos países. Sin embargo, Australia, Canadá, Estados Unidos y Reino Unido adoptaron su configuración electoral original en el siglo XIX, sin tener alteraciones significativas desde entonces. En un intervalo temporal menor, Finlandia mantiene su modelo de RP con voto preferencial desde 1906.

Considerando la distinción entre regímenes liberales y autocráticos oferecido por Polity IV, un número equivalente a 101 (49,7\%) de las reformas electorales aquí registradas fueron realizadas bajo sistemas competitivos, 63 (31\%) bajo regímenes autoritarios y 39 constituyeron las primeras instituciones electorales de nuevos Estados nacionales construidos a partir de procesos de descolonización o formación de nuevas naciones. Como el objetivo del trabajo consiste en evaluar la resistencia institucional para reformas electorales, en contextos poliárquicos, y delante de los problemas metodológicos resultantes de desprecio por las diferencias existentes en procesos de negociación para el cambio institucional en sistemas democráticos y autoritarios, se optó por concentrar el foco del análisis a los casos de reforma electoral promovidos dentro de sistemas competitivos.

El primer paso en esta dirección constituye razonablemente en un inventario de las reformas, según el período en que ocurrieron, tal como puede ser observado en la tabla 1. 
TABLA 1: Distribución de las reformas electorales según período y modelo adoptado

\begin{tabular}{llrrc}
\hline \multirow{3}{*}{ Reforma } & \multicolumn{4}{c}{ Período } \\
\cline { 2 - 5 } & \multicolumn{5}{c}{ hasta 1899 } & 1900 a 1945 & pos 1945 \\
\cline { 2 - 5 } & MAY & 90,9 & 26,1 & 15,4 \\
& MIX** & 4,5 & 4,3 & 43,6 \\
& LC*** & 4,5 & 52,2 & 23,1 \\
& VP**** & - & 17,4 & 17,9 \\
\hline
\end{tabular}

*MAY- mayoritario; **MIX- mixto; ***LC- lista cerrada; ****VP- voto preferencial.

Fuentes: Polity IV; ksghome.harvard.edu/ pnorris; idea.int; ipu.org; electoral-reform.org.uk; ifes.org; odci. gov/cia; Colomer (2004); Norris (2004); Rose (2000).

Cuando se considera el modelo de institución electoral adoptado en cada reforma y el período en que el cambio fue promovido, se puede constatar que hasta el final del siglo XIX, prácticamente todo el ejercicio de ingeniería electoral se movía en torno a sistemas mayoritarios de representación. Las únicas excepciones son el modelo mixto, adoptado en Portugal en 1896 después de un año de funcionamiento de un sistema mayoritario, y la fórmula de representación proporcional introducida originalmente en Bélgica en 1899 después de 68 años de operación de fórmula mayoritaria uninominal. Una óptica alternativa para capturar la popularidad de sistemas mayoritarios hasta el final del siglo XIX puede ser alcanzada cuando se verifica que 62,5\% (o 20 de las 32 ocurrencias) de todas las reformas que implementaron reglas mayoritarias se verificaron también dentro de aquel intervalo, en contexto que corresponde a las restricciones al sufragio universal masculino. Los mercados políticos regidos por el voto restringido en criterios de renta y propiedades, hasta mediados del siglo XIX, fueron dominio de "notables". Los candidatos - de la misma forma que sus electores- disponían de fortunas personales, diplomas, relaciones y reputaciones territoriales. En este momento la distancia social entre representante y representados es reducida (Gaxie, 1989), constituyendo transacciones virtualmente personalizadas y cara a cara. Las distancias ideológicas entre representantes son limitadas, no ofreciendo fundamento para alineamientos permanentes. Los electores son menos sensibles a discursos ideológicos que en ese momento, no son tan diferentes, sino más sensibles con el retorno material que la confianza prestada le pueda ofrecer (Gaxie, 1993). Las circunscripciones de mayoría uninominal parecen funcionales a un formato de competencia restricta en torno a candidatos con recursos de notoriedad personal. Sin embargo, a lo largo del siglo XX, acompañando la tendencia de ampliación de la competencia electoral, se verifica una constante caída en la opción por reglas electorales mayoritarias como opción cuando del ejercicio de reforma electoral se trata.

La primera mitad del siglo XX representa un contexto marcado por una fuerte inflexión en la dirección de la representación proporcional, constituyendo, por la agregación LC/VP más de $69 \%$ de los cambios electorales registrados en aquel período. En especial, es el contexto en que la opción por sistemas de representación proporcional con listas cerradas registra su edad de oro, constituyendo $52,2 \%$ de todas las reformas electorales promovidas entre 1900 y 1945 . La ampliación del sufragio introdujo en el mercado electoral un nuevo personal político, de origen social más bajo, que no disponía de prestigio individual, poseciones materiales y/o recursos de mando (Weber, 1984). En este sentido, estos nuevos actores podían ser miembros de las clases medias, como de los cuadros políticos del radicalismo en la Francia de la III República (Gaxie, 1993), o del mundo sindical, como en la socialdemocracia alemana. 
Para enfrentar la concurrencia de políticos tradicionales, es necesario recurrir al capital político "prestado" por el partido, permitiendo al aspirante compensar la carencia de atributos personales como notoriedad, diploma, riqueza (Gaxie y Offerlé, 1985), a través de la oferta de contrapartidas simbólicas como programas, visiones de mundo, valores éticos, banderas sociales, transferidos por la identificación partidaria (Offerlé, 1985).

Tornándose eficaces como interpelación electoral, rótulos como izquierda y derecha, socialdemocracia, democracia-cristiana, laborista, conservador, permiten una economía en los costos de información para el elector estructurar sus opciones electorales (Downs, 1957). Trascendiendo a la confianza, conocimiento y lealtad personal, la naturaleza de los vínculos que ligan a representantes y representados bajo RP/lista cerrada constituye una estructura de incentivos para la formación de identidades políticas estables, fundados en la percepción del sentido de pertenencia a una comunidad de valores o intereses compartidos.

El surgimiento del sistema proporcional y el mecanismo de lista cerrada contribuyeron para reforzar imágenes partidarias, al mismo tiempo en que consagran la impersonalidad de los mandatos parlamentarios (Manin, 1995). Sugerente es la observación de la sincronía entre la introducción del sufragio universal masculino -y, por lo tanto, una mutación en la naturaleza de las transacciones en el mercado electoral-y la adopción de reglas proporcionales: 1894 y 1899, en Bélgica; 1906 y 1906 en Finlandia; 1907 y 1907 en Suecia; 1907 y 1920 en Austria, 1919 en Italia; 1915 y 1918 en Dinamarca; 1917 en Holanda. No obstante, las razones que motivaron su adopción hayan variado en cada caso -el miedo de los conservadores con respecto al crecimiento y una eventual obtención de la mayoría por los socialistas, o la pretensión de éstos en garantizar la representación- el resultado es que bajo este formato electoral, y aún sometido a las condiciones de lista partidaria cerrada, el elector se vio frente a una opción impersonal y el candidato, sometido al control ejercido por la organización partidaria que, al final, pasa a definir quién puede ser candidato y cuál será su posición en la lista.

El período posterior a 1945 presentó un flujo importante en la dirección de fórmulas mixtas, que fueron implementadas en casi la mitad de las reformas introducidas bajo instituciones poliárquicas. Analizando la evidencia por otro lado, se verifica que 17 de los 19 cambios en dirección de sistemas mixtos fueron promovidos a partir de 1945, representando $89,5 \%$ de los casos de cambio proinstituciones mixtas.

A continuación, información relevante para la reconstitución de modelos de cambio electoral es ofrecida por el registro del flujo de cada reforma, considerándose el statu quo y la institución adoptada. La tabla 2 resume esta información:

TABLA 2: Flujo de reformas electorales conforme el origen

\begin{tabular}{llcccc}
\hline & \multicolumn{5}{c}{ Reforma } \\
\cline { 2 - 6 } Statu quo & MAY & MIX & LC & VP \\
& MAY* & 78,1 & 63,2 & 72,2 & 36,4 \\
& MIX** & 3,1 & - & 18,2 & 9,1 \\
& LC*** & 18,8 & 21,1 & - & 54,5 \\
& VP**** & - & 15,8 & 9,1 & - \\
\hline
\end{tabular}

*MAY- mayoritario; **MIX- mixto; ***LC- lista cerrada; ****VP- voto preferencial.

Fuentes: Polity IV; ksghome.harvard.edu/ pnorris; idea.int; ipu.org; electoral-reform.org.uk; ifes.org; odci. gov/cia; Colomer (2004); Norris (2004); Rose (2000). 
El statu quo constituido por instituciones mayoritarias responde por $68 \%$ del background previo a la introducción de nuevas reglas electorales bajo el régimen poliárquico, patrón que acompaña a la introducción de variaciones en los modelos mayoritarios (78,1\%), o a la introducción de fórmulas mixtas $(63,2 \%)$ o de representación proporcional con lista cerrada (72,2\%). Discrepancias en este patrón pueden ser encontrados en reformas que introducen modelos de representación proporcional con voto preferencial, cuando poco más de 1/3 de los cambios institucionales promovidos en esta dirección son implantados en sustitución a los sistemas mayoritarios. La mayor frecuencia de reformas, en este caso, está relacionada al cambio de modelos de lista dentro de fórmulas de representación proporcional (54,5\%), alterando la lista cerrada por procedimientos de competencia intrapartidaria por votos preferenciales.

Una visión distinta para analizar el proceso de reformas electorales puede ser ofrecida cuando se recupera la información relativa al resultado de los cambios, tal como puede ser verificado en la tabla 3.

TABLA 3: Flujo de reformas electorales conforme el resultado

\begin{tabular}{lccccc}
\hline & \multicolumn{5}{c}{ Resultado } \\
\cline { 2 - 6 } Statu quo & MAY & MIX & LC & VP \\
& MAY* & 43,9 & 21,1 & 28,1 & 7,0 \\
& MIX** & 16,7 & - & 66,7 & 16,6 \\
& LC*** & 37,5 & 25,0 & - & 37,5 \\
& VP*** & - & 60,0 & 40,0 & - \\
\hline
\end{tabular}

*MAY- mayoritario; **MIX- mixto; ***LC- lista cerrada; ****VP- voto preferencial.

Nota: En este caso fueron considerados cambios entre instituciones mayoritarias, como plurality/runoff.

Fuentes: Polity IV; ksghome.harvard.edu/ pnorris; idea.int; ipu.org; electoral-reform.org.uk; ifes.org; odci. gov/cia; Colomer (2004); Norris (2004); Rose (2000).

Considerándose un continuo fijado con base en el grado de competencia ofrecido por el statu quo MAY-MIX-LC-VP (Colomer, 2004), se verifica que 70,3\% de las reformas electorales obedece a un patrón contiguo. Las fórmulas mayoritarias fueron sustituidas, predominantemente, por variaciones de regla de mayoría, o por la creación de reglas mixtas. Los casos más frecuentes de desvío en relación a este patrón ocurren cuando se da el cambio a partir de un statu quo mayoritario para LC (16 casos), o en dirección inversa LC hacia MAY (no obstante, en este flujo restricto sólo a seis casos). Estos desvíos pueden ser comprendidos cuando se identifica el intervalo temporal del cambio. Hasta la primera mitad del siglo XX, la sustitución de statu quo mayoritario por representación proporcional con lista cerrada (LC) constituía la alternativa más probable como resultado de la propia escasez de alternativas mixtas o de RP con voto preferencial.

\section{V. ¿IMPORTA EL TIEMPO?}

Además de la dirección de los cambios, parece relevante considerar el efecto producido por el tiempo en el incremento a los costos de reversión para el statu quo institucional. Las interpretaciones fundadas en la comprensión de procesos políticos como condicionados por modelos path-dependent sugieren que el prolongamiento temporal de una estructura institucional debe generar efecto de 
"retornos crecientes" (North, 1990; Pierson, 2004): la repetición de las reglas implica una rutina institucional, reduciendo los costos de transacción presentes en las interacciones y en el juego político, moldeando estrategias de los agentes a los impedimentos y oportunidades definidos por las instituciones establecidas, al mismo tiempo que amplía el riesgo y la incertidumbre en cuanto a alteraciones. Los políticos electos por determinada fórmula electoral tendrían siempre resistencia en alterar esas reglas, una vez que esa alternativa pudiera invertir las condiciones de la competencia electoral, alterando sus prospectivas de manutención/movilidad de la carrera política. Cuanto más se prolonga el tiempo de vida de cada modelo institucional, mayores son los costos de transacción necesarios para la promoción de una coalición procambio -o en el lenguaje de Tsebelis (2002), menor el objetivo de cambios- indicando presencia de asociación negativa entre tiempo y probabilidad de reforma institucional.

Se buscó medir esta premisa, considerando el tiempo de vida del statu quo electoral, previo a cada reforma institucional, o sea, la duración en años de un modelo de regla electoral antes de su sustitución. Los casos fueron divididos en tres grupos: aquellos en que la reforma ocurre hasta 15 años de funcionamiento de las reglas anteriores; reformas verificadas en el intervalo entre 16 y 30 años de vida del modelo previo y, finalmente, reformas implementadas después de un largo período de operación del statu quo, superior a 30 años de duración. La distribución de los casos es presentada en la figura 1.

FIGURA 1: Tiempo de duración del statu quo antes de la reforma electoral (en \%)

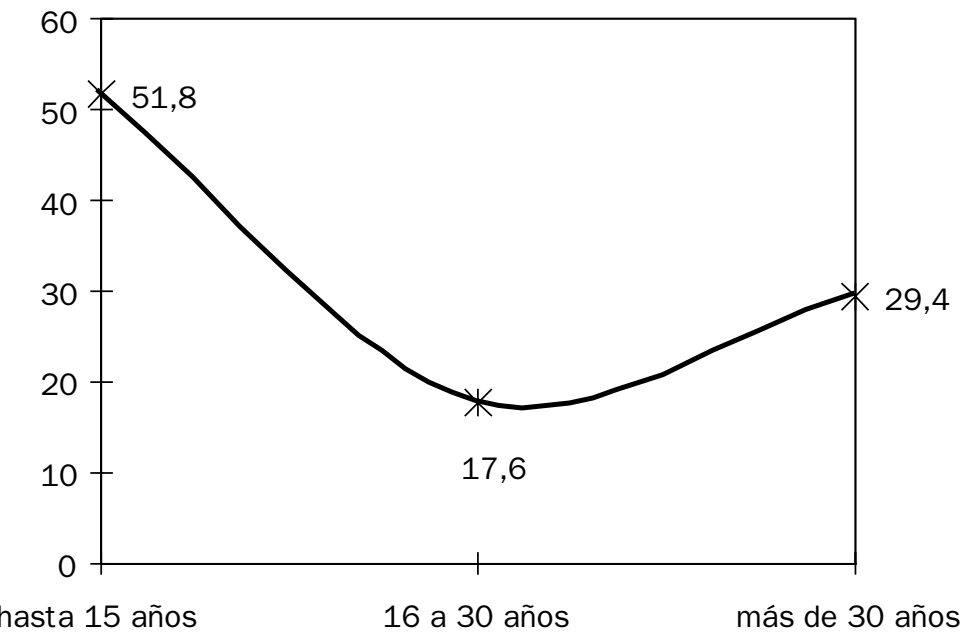

Conforme a lo esperado, la mayor frecuencia de reformas electorales se verifica en un intervalo de tiempo corto, después de la implantación del statu quo previo. 0 sea, las reglas institucionales presentan mayor riesgo de ruptura, cuando más recientes sean o menos tiempo tengan en operación. En este caso, los costos de transacción para los cambios probablemente sean menores, los procesos de reclutamiento político aún no hayan seleccionado una generación de candidatos con estrategias enteramente encuadradas bajo las nuevas oportunidades y los riesgos con un nuevo 
cambio sean más modestos. Conforme a lo previsto, en la medida que el tiempo de duración de las nuevas reglas es extendido para un intervalo entre 16 y 30 años, la frecuencia de cambios en las reglas electorales declina dramáticamente, indicando una estructura de oportunidades en que probablemente la configuración institucional ya se haya establecido, haya sido incorporada a las estrategias de los competidores, y los nuevos competidores sean los producidos por las fórmulas en vigor.

Sin embargo, la sorpresa se da con el incremento verificado en la frecuencia de reformas electorales en instituciones dotadas de períodos más largos (arriba de 30 años) de existencia. Aunque lo que debiera ocurrir es que, habiendo alcanzado una duración larga, la probabilidad de cambio fuera aún menor, ello no se da de esa forma. Así, la premisa sobre que las instituciones en operación producen efectos de retornos temporales crecientes, ampliando los costos de reversión y adquiriendo condición path-dependent, debe incorporar la complejidad presente en esta situación, identificando las contingencias que limitan el plazo de validez institucional. 0 sea, incluso después de su institucionalización, las organizaciones no parecen resistir a factores-endógenos o contextuales- que alteren la eficacia de las reglas para generar equilibrio, induciendo a los agentes a nuevos cálculos de premios y recompensas, con beneficios consecuentes del cambio institucional. La próxima sección de este trabajo retomará este problema, intentando aislar factores diferenciales que pueden afectar la estabilidad de cada fórmula electoral, según la duración de cada uno de los intervalos temporales y, especialmente, identificar las condiciones que pueden contribuir para el quiebre del equilibrio institucional después de larga durabilidad anterior.

Antes de eso, se pretende identificar si existen intervalos temporales distintos, según cada flujo de cambio institucional. La tabla ofrece la información sobre el tiempo medio de prolongamiento del statu quo, en relación a la alternativa institucional adoptada:

TABLA 4: Intervalo temporal entre adopción statu quo y reforma electoral según modelos electorales (en años)

\begin{tabular}{llrrrrr}
\hline & \multicolumn{7}{c}{ Reforma } \\
\cline { 2 - 7 } Status quo & MAY* & 21 & 23 & 29 & 41 & 25 \\
& MIX** & 5 & - & 12 & 3 & 9 \\
& LC*** & 7 & 21 & - & 39 & 23 \\
& VP**** & - & 16 & 8 & - & 13 \\
\hline
\end{tabular}

*MAY- mayoritario; **MIX- mixto; ***LC- lista cerrada; ****VP- voto preferencial.

Fuentes: Polity IV; ksghome.harvard.edu/ pnorris; idea.int; ipu.org; electoral-reform.org.uk; ifes.org; odci. gov/cia; Colomer (2004); Norris (2004); Rose (2000).

La continuidad temporal del statu quo parece relacionada a la distancia de la alternativa de reforma, en relación a las reglas o al formato de la competencia electoral anterior. Así, el cambio en las instituciones electorales puede ser más probable, y abreviar la duración de la fórmula antigua, cuando las diferencias entre statu quo y winset fueran menores, indicando un patrón predominante de cambio dentro de una matriz institucional original. Cuando el statu quo es representado por fórmulas electorales mayoritarias, su durabilidad temporal aumenta progresivamente a medida 
que la alternativa de reforma sea ofrecida dentro del continuo MAY-MIX-LC-VP, correspondiendo a los márgenes de competencia permitidos a los partidos y candidatos, o al objetivo de opción ofrecido a los electores. Las fórmulas de representación proporcional con voto preferencial son adoptadas solamente después de largos períodos de funcionamiento de reglas mayoritarias o proporcionales de lista cerrada. Lo inverso no parece cierto: el cambio por sistemas menos inclusivos puede ocurrir posterior a intervalos temporales más limitados, como en el pasaje LC hacia MAY. La aparente discrepancia presentada por la reforma MAY-MAY, con tiempo medio de 21 años, no contradice la naturaleza gradual que parece caracterizar la adopción de cambios en las reglas electorales. Por último, se debe destacar el precario equilibrio representado por fórmulas mixtas, separados por cortos intervalos temporales en relación a la introducción de alternativas electorales.

Por otro lado, los tiempos medios de persistencia del statu quo presentados anteriormente no deben ser confundidos con la durabilidad temporal de cada modelo electoral. En los datos presentados hasta aquí se consideró apenas el intervalo de tiempo de operación entre una determinada fórmula electoral y su sustitución por otro sistema. Por lo tanto, cada modelo fue considerado solamente cuando fue hecha la permuta por otra alternativa. Igualmente, un gran número de casos conocieron el equilibrio, después del cambio institucional en un punto más o menos distante en el pasado. Para corregir esta distorsión, fueron incorporados los tiempos relativos a los cambios que produjeron equilibrios estables, o sea, casos en que una vez introducida la reforma, la institución fue mantenida hasta el presente. Este procedimiento permite una medida aproximada de los valores medios de duración de cada una de las reglas electorales en examen, conforme presentado en la figura 2.

FIGURA 2: Duración temporal media en diferentes modelos electorales (media, en años)

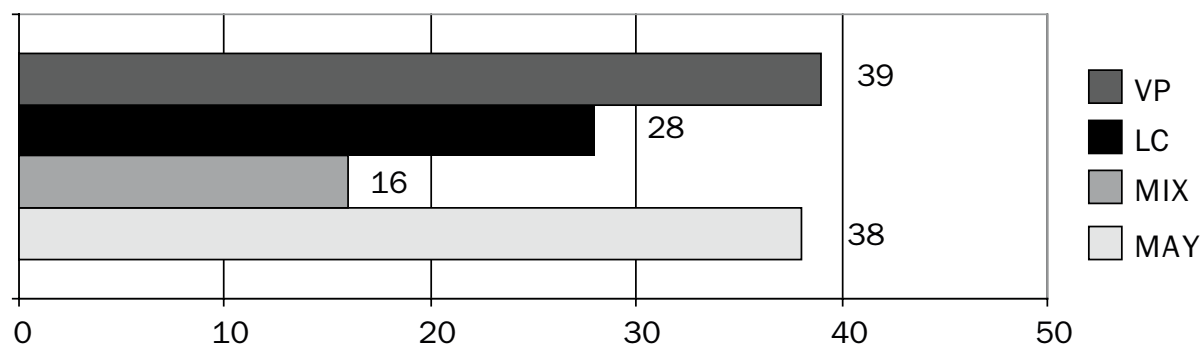

MAY- mayoritario; MIX- mixto; LC- lista cerrada; VP- voto preferencial.

Fuentes: Polity IV; ksghome.harvard.edu/ pnorris; idea.int; ipu.org; electoral-reform.org.uk; ifes.org; odci. gov/cia; Colomer (2004); Norris (2004); Rose (2000).

Los cinco casos de representación proporcional con voto preferencial que fueron sustituidos por RP/lista cerrada o fórmula mixta y presentaron tiempo de vida breve, simbolizan una excepción dentro del grupo de instituciones con esa configuración electoral. En su conjunto, las democracias que adoptan RP/voto preferencial presentaron los más elevados tiempos medios de duración. Finlandia (88), Irlanda (83), Uruguay (71), Brasil (59) son ejemplos de configuraciones en equilibrio estable, traducido en elevada continuidad temporal. Los sistemas mayoritarios presentan, 
igualmente, patrones estables, en niveles equivalentes a los de VP. Estados Unidos, Reino Unido, Canadá y Australia, con instituciones electorales formadas en el siglo XIX o, en el límite, en el inicio del XX, contribuyen para definir esta propiedad de las instituciones mayoritarias. En el extremo opuesto, fórmulas mixtas revelan continuidades institucionales más modestas, alcanzando apenas 16 años de duración media. Entre los factores que pueden haber contribuido para esta precariedad, se debe considerar que 26 de las 31 reformas con adopción de este modelo ocurrieron después de 1945 y, de forma más saliente 19, solamente posterior a 1990. Por otro lado, una hipótesis que podría merecer ser sometida a pruebas más rigorosas sería la asociación entre la constitución híbrida de este sistema, con dos distintas estructuras de incentivos (mayoritaria y proporcional) para carreras políticas, y la inducción a estrategias de defección en relación a las reglas, fragilizando el equilibrio institucional existente.

A continuación, se intentó explorar aquellas variables que pueden influenciar la promoción de reformas electorales. Para examinar si la ocurrencia de cambios en las reglas electorales obedece a modelos convergentes o diferenciados, según el intervalo de tiempo previo del statu quo, fueron pasadas tres series de regresión logística, considerando reformas electorales en plazo corto (hasta 15 años), medio (de 16 a 30 años) o largo (arriba de 30 años). Fue definida como variable dependiente, una dummy (1 para años con reformas, 0 para años sin reformas) referentes a la ocurrencia de cambios electorales. Las variables independientes escogidas fueron la variación anual en el producto interno bruto per cápita (PIB), competencia electoral, según Polity IV (COMPET) y el tiempo de funcionamiento del régimen democrático (INSTIT). Los resultados están contenidos en la tabla de abajo, que presenta coeficientes B, significancia y error-estandarizado (entre paréntesis).

TABLA 5: Regresión logística: reformas conforme tiempo previo en relación a las variables escogidas (B, sig, S.E.)

\begin{tabular}{lcccc}
\hline & \multicolumn{4}{c}{ REFORMAS ELECTORALES } \\
\cline { 2 - 5 } & PIB & $\begin{array}{c}\text { Reformas después } \\
\text { statu quo corto }\end{array}$ & $\begin{array}{c}\text { Reformas después } \\
\text { statu quo medio }\end{array}$ & $\begin{array}{c}\text { Reformas después } \\
\text { statu quo largo }\end{array}$ \\
\hline Variables & $-0.110 * * *$ & -0.019 & -0.039 \\
independientes & $(0.027)$ & $(0.106)$ & $(0.045)$ \\
& COMPET & $0.181^{*}$ & 0.250 & $0.570 * *$ \\
& & $(0.041)$ & $(0.199)$ & $(0.140)$ \\
& \multirow{2}{*}{ INSTIT } & $-0.043^{* *}$ & 0.001 & 0.003 \\
& & $(0.013)$ & $(0.020)$ & $(0.009)$ \\
\hline
\end{tabular}

*** sig a $1 \% * *$ sig a $5 \% *$ sig a $10 \%$.

Fuentes: Polity IV; ksghome.harvard.edu/ pnorris; idea.int; ipu.org; electoral-reform.org.uk; ifes.org; odci. gov/cia; Colomer (2004); Norris (2004); Rose (2000).

Las reformas electorales implementadas posteriormente al reciente período de operación del statu quo electoral pueden ser afectadas por una combinación de factores resultantes del desempeño económico, incremento en la competencia electoral y una precaria institucionalización del régimen 
democrático. La continuidad/cambio en las reglas electorales parece sensible al desempeño de la economía, medido conforme la variación anual del PIB. Las reformas producidas después de un intervalo corto de duración de las reglas electorales anteriores presentan tasas medias negativas de crecimiento del PIB nacional $(-4,46 \%)$, en contraste con los valores medios de los años sin reformas $(0,65 \%)$. La misma relación no ocurre en el grupo de las reformas posterior al tiempo medio (1,33\% x -2,47 \%) o largo (0,48\% x -3,67\%), cuando el desempeño económico en los años con reformas es mayor y positivo. De otro lado, la variación media negativa es en años sin reformas. Esto parece indicar que las instituciones electorales de implantación reciente son sensibles al desempeño económico, sucumbiendo cuando este es negativo. Sin embargo, después de intervalos mayores de funcionamiento (probablemente a partir de 15 años), las reglas electorales adquieren resistencia al contagio de la economía, pudiendo ser reformadas, pero en este caso, debido a factores que no dependen de crisis económicas.

La supervivencia de fórmulas electorales a corto plazo está asociada a la longevidad previa de las instituciones poliárquicas, lo que implica que disminuye la probabilidad de reformas sucesivas en la medida en que regímenes democráticos se institucionalizan. Inversamente, el incremento en la competencia electoral afecta las posibilidades de introducción de reformas en las reglas electorales, después de un breve período de experimentación de las fórmulas vigentes.

Finalmente, la única variable con asociación de estadística significativa (al 5\%) con reformas electorales posteriores a 30 años o más de funcionamiento de las reglas previas es la "competencia electoral", según el indicador de Polity IV. Lo que puede sugerir que los "retornos crecientes"” ofrecidos por el tiempo y por la rutina institucional, si pueden sobrevivir a ciclos económicos, parecen menos elásticos al incremento en la competencia, traducida en mayor fragmentación política y electoral.

A continuación, parece necesario probar la relación entre los modelos electorales previos e intervalos temporales de duración del statu quo. Se trata, en otras palabras, de verificar si existen probalidades distintas de duración (corta/media/larga) de cada institución. Esta información puede ser verificada en la tabla 6.

TABLA 6: Intervalo de tiempo en relación al statu quo electoral

\begin{tabular}{lrrrrrr}
\hline & \multicolumn{2}{c}{ Corto } & \multicolumn{2}{c}{ Medio } & \multicolumn{2}{c}{ Largo } \\
\hline MAY* & 47,4 & $(27)$ & 21,0 & $(12)$ & 31,6 & $(18)$ \\
MIX** & 83,3 & $(5)$ & - & & 16,7 & $(1)$ \\
LC*** & 50,0 & $(8)$ & 18,7 & $(3)$ & 31,2 & $(5)$ \\
VP**** & 80,0 & $(4)$ & - & & 20,0 & $(1)$ \\
\hline
\end{tabular}

*MAY- mayoritario; **MIX- mixto; ***LC- lista cerrada; ****VP- voto preferencial.

Fuentes: Polity IV; ksghome.harvard.edu/ pnorris; idea.int; ipu.org; electoral-reform.org.uk; ifes.org; odci. gov/cia; Colomer (2004); Norris (2004); Rose (2000).

Los modelos de representación proporcional con voto preferencial (VP) y las fórmulas mixtas (MIX) presentan mayor probabilidad de ser sustituidos por reglas alternativas, en los primeros años de su implantación. Las únicas excepciones fueron representadas por la sustitución de la 
fórmula mixta por mayoritaria en Portugal (1915) y la reforma del sistema proporcional con voto preferencial por el sistema mixto en Italia en 1993. En el caso de la reforma italiana, la sustitución del voto preferencial puede ser comprendida como resultado del contexto de colapso de la partitocrazia peninsular activada por el impacto de denuncias de corrupción y al involucramiento con redes ilegales. Esto produjo una fuerte retracción en su organización más tradicional, la democracia-cristiana, la virtual desaparición de los socialistas y la eclosión de nuevas corrientes, como Forza Italia, provocando el realineamiento político con impactos sobre estrategias de carrera y discontinuidad en el reclutamiento parlamentario. Habiéndose mantenido a un nivel próximo de $35 \%$ desde 1953, la renovación en los escaños parlamentarios alcanza la marca de $70 \%$ en las elecciones para la legislatura 1994/96 (Briquet, 1999; Bartolini y D’Alimonte, 1995). Esta cifra se debe asignar a dos condiciones que contribuyeron a aumentar las posibilidades electorales de novatos en la carrera política. En primer lugar, la sanción proferida por el elector. Simultáneamente, la defección de un número significativo de veteranos en recandidatearse, anticipándose a una probable derrota electoral puede haber contribuido para facilitar la conquista de los escaños por nuevos desafiantes (Briquet, 1999: 257). De hecho, el índice de recandidatura de los entonces parlamentarios en 1994 corresponde al 49\%, inferior a los 65\% de 1987 y 54\% de 1992, y más revelador es todavía entre aquellos que se representan, apenas $21 \%$ obtuvieron éxito en conservar su escaño. La evasión llega de forma desigual a las principales corrientes: más fuerte entre los democristianos (63\%) que en los ex comunistas (34,6\%), de la misma forma que la confirmación del mandato es obtenida por apenas $12 \%$ de los candidatos del PPI, contra cerca de mitad entre los ex comunistas. La lectura de esta información parece evidente: la única reforma de modelo proporcional con voto preferencial, después de un largo intervalo de duración previa, parece haber sido resultado de la fuerte crisis que alcanza a la elite política y legislativa italiana traducida en un aumento en los índices de evasión parlamentaria. Sin expectativas de continuidad en la carrera legislativa ("ambición estática"), los diputados se tornan indiferentes en relación a la incertidumbre generada por un cambio en las reglas electorales, neutralizando los efectos de inercia provocados por la larga duración temporal de las mismas instituciones.

Las reglas electorales mixtas y de representación proporcional con voto preferencial producen, entre otros efectos, un incremento en el costo de información para el elector definir la dirección de su voto. Sistemas mixtos, por su estructura híbrida, que envuelve una duplicación de candidaturas presentadas bajo distintas reglas electorales mayoritarias y proporcionales, tornan más compleja la comprensión de los procedimientos de conversión de votos en escaños y a la propia oferta de candidaturas. Si la representación proporcional acostumbra a reducir los incentivos para el voto estratégico, y con ello, aumentar la dispersión en la oferta electoral (Cox, 1997), la representación proporcional con voto preferencial tiende a ser acompañada por un mayor número de partidos efectivos, cuando la comparamos con modelos proporcionales de lista cerrada (Marenco dos Santos, 2005). Esto representa, igualmente, ampliación en los costos de información electoral para los votantes, una vez que mayor número de leyendas significa mayor complejidad para discernir el contenido específico de sus rótulos y la "clareza de responsabilidad" (Powell, 1990) gubernamental de cada una. Sin embargo, además de incrementar el número de leyendas nominales, instituciones proporcionales de voto preferencial ofrecen oportunidad para la presentación de candidaturas personalizadas, a disputar preferencias intrapartidarias junto al electorado. Más partidos y, sobre todo, voto preferencial dentro de cada partido constituyen un 
incremento sustantivo en el volumen de información exigido al elector para que éste decida cómo conferir su sufragio. De la misma forma, en este contexto, organizaciones partidarias disponen de recursos escasos para controlar el ingreso y movilidad en carreras políticas, o seleccionar el acceso a cargos y puestos entre candidatos individuales. En períodos de implantación reciente -especialmente cuando va acompañado de factores exógenos perjudiciales como desempeño económico negativo- es de esperarse que el apoyo a estas reglas sea más precario, ya sea por electores o por dirigentes partidarios. No obstante, la continuidad del tiempo representa una oportunidad para la economía en los costos de información electoral, permitiendo que electores conviertan reputación personalizada (inducida por el voto preferencial) en reputación partidaria. En otro trabajo (Marenco dos Santos, 2005) fue posible aislar una relación entre el tiempo medio de vida de las organizaciones partidarias y las tasas de volatilidad electoral: países en que el tiempo medio de vida de los partidos es más elevado, los niveles de volatilidad del voto son más reducidos, confirmando el efecto de positive feedback promovido por lo rutinario de la competencia electoral. Por otro lado, la inclusividad ofrecida por el voto preferencial se traduce en un aumento en la presencia de veto-players (facciones y lemas partidarios, candidaturas individuales) para los cuales el cambio del statu quo representa una alternativa de una gran incertidumbre. Ello quiere decir que, con el tiempo, los modelos de representación proporcional con voto preferencial constituyen un modelo de improbable defección.

\section{CONCLUSIÓN}

En las últimas dos décadas la agenda de la ciencia política internacional permitió desarrollos teóricos y resultados empíricos capaces de demostrar que las instituciones son relevantes, condicionando estrategias y resultados políticos. Sin embargo, una laguna importante todavía reside en el desarrollo de una "teoría de las instituciones", según Diermeier y Krehbiel, capaz de volcar su foco para la explicación de las condiciones responsables por la formación institucional, y en qué medida opciones, estrategias y contextos interfieren en el diseño de instituciones, que, bien sabemos, después de creadas irán a moldear opciones, estrategias y contextos. Este trabajo intentó explorar esa laguna, analizando las condiciones que limitan la opción de instituciones electorales. Fue posible identificar: [1] la presencia de tendencias que asociaron la adopción de fórmulas electorales mayoritarias al siglo XIX, de representación proporcional en la primera mitad, e instituciones mixtas, en la segunda mitad del siglo XX; [2] instituciones electorales mayoritarias como statu quo presente en cerca de 2/3 de las reformas electorales verificadas; [3] la predominancia de un patrón contiguo en los cambios electorales promovidos en instituciones electorales poliárquicas; [4] la mayor probabilidad para la ocurrencia de reformas, cuanto menor sea el tiempo de operación del statu quo electoral; [5] incremento en la competencia electoral como factor responsable por la reducción en la resistencia de fórmulas electorales ya institucionalizadas. Una agenda de estudios y futuras investigaciones sobre opción institucional deberá avanzar en la comprensión sobre los efectos ejercidos por el tiempo sobre la resistencia y retroalimentación institucional, así como influencias contextuales representadas por las ondas coyunturales de cambio y el impacto provocado por el incremento en la competencia política sobre la supervivencia de diferentes modelos institucionales. 


\section{ANEXO}

TABLA 7: Reformas electorales en contextos competitivos

\begin{tabular}{|c|c|c|c|}
\hline Año & País & Statu quo & Reforma \\
\hline 1820 & Francia & MAY & MAY \\
\hline 1842 & Estados Unidos & MAY & MAY \\
\hline 1848 & Francia & MAY & MAY \\
\hline 1854 & España & MAY & MAY \\
\hline 1857 & España & MAY & MAY \\
\hline 1859 & Portugal & MAY & MAY \\
\hline 1865 & España & MAY & MAY \\
\hline 1875 & Francia & MAY & MAY \\
\hline 1878 & España & MAY & MAY \\
\hline 1879 & Chile & MAY & MAY \\
\hline 1882 & Italia & MAY & MAY \\
\hline 1885 & Francia & MAY & MAY \\
\hline 1885 & Reino Unido & MAY & MAY \\
\hline 1886 & Colombia & MAY & MAY \\
\hline 1887 & Holanda & MAY & MAY \\
\hline 1889 & Francia & MAY & MAY \\
\hline 1890 & Brasil & MAY & MAY \\
\hline 1892 & Brasil & MAY & MAY \\
\hline 1892 & Italia & MAY & MAY \\
\hline 1895 & Portugal & MAY & MAY \\
\hline 1896 & Portugal & MAY & MIX \\
\hline 1899 & Bélgica & MAY & LC \\
\hline 1901 & Portugal & MIX & MAY \\
\hline 1910 & Colombia & MAY & MAY \\
\hline 1912 & Argentina & MAY & MAY \\
\hline 1915 & Portugal & MIX & LC \\
\hline 1915 & Dinamarca & MAY & VP \\
\hline 1918 & Holanda & MAY & $\mathrm{VP}$ \\
\hline 1919 & Bélgica & LC & $\mathrm{VP}$ \\
\hline 1919 & Italia & MAY & $\mathrm{VP}$ \\
\hline 1919 & Francia & MAY & LC \\
\hline 1919 & Alemania & MAY & LC \\
\hline 1920 & Dinamarca & VP & LC \\
\hline 1921 & Noruega & MAY & LC \\
\hline 1922 & Lituania & MAY & LC \\
\hline 1923 & Italia & VP & MIX \\
\hline 1925 & Chile & MAY & LC \\
\hline 1926 & Grecia & MAY & LC \\
\hline 1927 & Francia & LC & MAY \\
\hline 1928 & Grecia & LC & MAY \\
\hline 1931 & Colombia & MAY & LC \\
\hline
\end{tabular}




\begin{tabular}{|c|c|c|c|}
\hline 1932 & Grecia & MAY & LC \\
\hline 1933 & Grecia & LC & MAY \\
\hline 1933 & Perú & MAY & LC \\
\hline 1936 & Grecia & MAY & LC \\
\hline 1951 & Colombia & LC & MIX \\
\hline 1952 & Grecia & LC & MAY \\
\hline 1953 & Italia & VP & MIX \\
\hline 1953 & Filipinas & MAY & MAY \\
\hline 1953 & Dinamarca & LC & VP \\
\hline 1953 & Costa Rica & MIX & LC \\
\hline 1956 & Italia & MIX & VP \\
\hline 1956 & Grecia & MAY & MIX \\
\hline 1958 & Grecia & MIX & LC \\
\hline 1958 & Francia & LC & MAY \\
\hline 1963 & México & MAY & MIX \\
\hline 1966 & Honduras & MAY & LC \\
\hline 1970 & Austria & LC & VP \\
\hline 1970 & Suecia & LC & VP \\
\hline 1978 & Sri Lanka & MAY & VP \\
\hline 1985 & Noruega & LC & VP \\
\hline 1986 & Francia & MAY & LC \\
\hline 1988 & Francia & LC & MAY \\
\hline 1989 & Venezuela & LC & MIX \\
\hline 1991 & Bulgaria & MIX & LC \\
\hline 1992 & Albania & MAY & MIX \\
\hline 1992 & Estonia & LC & VP \\
\hline 1992 & Mongolia & MAY & MAY \\
\hline 1993 & Rusia & MAY & MIX \\
\hline 1993 & Moldava & MAY & LC \\
\hline 1993 & Italia & VP & MIX \\
\hline 1993 & Nueva Zelandia & MAY & MIX \\
\hline 1994 & Ucrania & MAY & MIX \\
\hline 1994 & Bolivia & LC & MIX \\
\hline 1994 & Japón & MAY & MIX \\
\hline 1995 & Armenia & MAY & MIX \\
\hline 1995 & Filipinas & MAY & MIX \\
\hline 1996 & Mongolia & MAY & MAY \\
\hline 1997 & Tailandia & MAY & MIX \\
\hline 1998 & Madagascar & LC & MIX \\
\hline 2000 & Croacia & MIX & LC \\
\hline 2001 & Polonia & VP & LC \\
\hline
\end{tabular}

MAY- mayoritario; MIX- mixto; LC- lista cerrada; VP- voto preferencial. MAY-MAY son equivalentes a cambios internos en instituciones mayoritarias, de uninominal a plurinominal y plurality a runoff.

Fuentes: Polity IV; ksghome.harvard.edu/ pnorris; idea.int; ipu.org; electoral-reform.org.uk; ifes.org; odci. gov/cia; Colomer (2004); Norris (2004); Rose (2000). 


\section{REFERENCIAS}

Bartolini, Stefano y Roberto D’Alimonte. 1995. "Les elections parlamentaires de 1994 en Italie. Competition majoritaire et réalignement partisan”. Revue Française de Science Politique 45 (6): 915-954.

Blais, André, Robert Young y Martin Turcotte. 2005. "Direct or Indirect? Assessing Two Approaches to the Measurement of Strategic Voting". Electoral Studies 24 (2): 163-346.

Boix, Carles. 1999. "Setting the Rules of the Game: The Choice of Electoral Systems in Advanced Democracies". American Political Science Review 93 (3): 609-624.

Briquet, Jean-Louis. 1999. “L'impératif du changement. Critique de la classe politique et renouvellement des parlamentaires dans la crise italienne (1992-1994)". En La Profession Politique, editado por Michel Offerlé. Paris: Belin, 255-277.

Cain, Bruce, John Ferejohn y Morris Fiorina. 1987. The Personal Vote: Constituency Service and Electoral Independence. Cambridge: Harvard University Press.

Carey, John y Matthew Shugart. 1996. "Incentives to Cultivate a Personal Vote: A Rank Ordering of Electoral Formulas". Electoral Studies 13 (4): 417-439.

Colomer, Josep. 2003. “It's Parties that Choose Electoral Systems (or Duverger's Laws Upside Down)". Paper presentado en la American Political Science Association Annual Meeting, Philadelphia.

Colomer, Josep (ed). 2004. Handbook of Electoral System Choice. New York and London: Palgrave-Macmillan.

Cox, Gary. 1997. Making Votes Count: Strategic Coordination in the World's Electoral Systems. Cambridge: Cambridge University Press.

Crisp, Brian et al. 2004. "Vote-Seeking Incentives and Legislative Representation in Six Presidential Democracies". The Journal of Politics 66 (3): 823-846.

Diermeier, Daniel y Keith Krehbiel. 2003. “Institutionalism as a Methodology”. Journal of Theoretical Politics 15 (2): 123-144.

Downs, Anthony. 1957. An Economic Theory of Democracy. New York: Harper \& Row.

Duverger, Maurice. 1954. Os partidos políticos. Brasília: UNB.

Duverger, Maurice. 1986. “Duverger's Law: Forty Years Later”. En Electoral Laws and their Political Consequences, editado por Bernard Grofman y Arend Lijphart. New York: Agathon, 69-84.

Fisichella, Domenico. 1984. "The Double-Ballot System as a Weapon against Anti-System Parties". En Choosing an Electoral System, editado por Arend Lijphart y Bernard Gorfman. New York: Praeger: 181-189.

Gaxie, Daniel. 1989. Explication du vote: un bilán des études électorales en France. Paris : Presses de la Fondation Nationale de Science Politique.

Gaxie, Daniel. 1993. La Démocratie Representative. Paris : Montchrestien.

Gaxie, Daniel y Michael Offerlé. 1985. "Les militants syndicaux et associatifs au pouvoir. Capital collectif et carrière politique”. En Les Élites Socialistes au Pouvoir, 1981-1985, editado por Pierre Birnbaun. Paris: Presses Universitaires de France, 105-138.

Grofman, Bernard. 2005. "Comparisons among Electoral Systems: Distinguishing Between Localism and Candidate-Centered Politics". Electoral Studies 24 (4): 735-740.

Guzzini, Stefano. 1994. "La longue nuit de la premiére république: I'implosion cliénteliste en Italie". Revue Française de Science Politique 44 (6): 979-1013.

Hall, Peter y Rosemary Taylor. 1996. "Political Science and the Three 'New Institutionalisms'”. Political Studies 44 (5): 936-957.

Le Duc, Lawrence, Richard Niemi y Pippa Norris. 1996. Comparing Democracies. Elections and Voting in Global Perspective. Thousand Oaks: Sage Publications.

Lijphart, Arend. 1990. "The Political Consequences of Electoral Laws, 1945-85". American Political Science Review 84 (2): 481-497.

Lijphart, Arend y Bernard Grofman. 1984. Choosing an Electoral System. Issues and Alternatives. New York: Praeger.

Lijphart, Arend. 1996. "The Puzzle of Indian Democracy: A Consociational Interpretation". American Political Science Review 90 (2): 258-268.

Lijphart, Arend. 1999. Patterns of Democracy: Government, Forms and Performance in Thirty-Six Countries. New Haven: Yale University Press.

Manin, Bernard. 1995. Principles of Representative Government. Cambridge: Cambridge University Press.

Marenco Dos Santos, André. 2005. "Modelos de lista eleitoral em perspectiva comparada". Paper presentado en el XXIX Encontro Anual da ANPOCS, Caxambú.

Moe, Terry. 1990. "The Politics of Structural Choice: Toward a Theory of Public Bureaucracy". En Organization Theory, editado por Oliver Williamson. Oxford: Oxford University Press, 116-142.

Norris, Pippa. 2002. Democratic Phoenix: Reinventing Political Activism. New York: Cambridge University Press. 
Norris, Pippa. 2004. Electoral Engineering. Voting Rules and Political Behavior. Cambridge: Cambridge University Press. North, Douglass. 1990. Institutions, Institutional Change and Economic Performance. Cambridge: Cambridge University Press.

Offerlé, Michael. 1985. "Mobilization électorale et invenction du citoyen: I'exemple du milieu urbain français à la fin du XIX siécle". En Explication du vote: un bilán des études électorales en France, editado por Daniel Gaxie. Paris: Presses FNSP, 127-148.

Ostrom, Elinor. 1990. Governing the Commons. The Evolution of Institutions for Collective Action. Cambridge: Cambridge University Press.

Peters, B. Guy. 1999. Institutional Theory in Political Science: The New Institutionalism. London: Cassel.

Pierson, Paul. 2004. Politics in Time. History, Institutions and Social Analysis. Princeton: Princeton University Press.

Rae, Douglas. 1967. The Political Consequences of Electoral Laws. New Haven: Yale University Press.

Rose, Richard. 2000. International Encyclopedia of Elections. Washington: Congressional Quarterly Press.

Sartori, Giovanni. 1996. Engenharia constitucional. Como mudam as constituições. Brasília: Unb.

Schepsle, Kenneth. 1995. "Studying Institutions: Some Lessons from the Rational Choice Approach". En Political Science in History. Research Programs and Political Traditions, editado por James Farr, John Dryzek y Stephen Leonard. Cambridge: Cambridge University Press, 276-295.

Taagepera, Rein. 1984. "The Efect of District Magnitude and Proprieties of Two-Seat Districts”. En Choosing an Electoral System, editado por Arend Lijphart y Bernard Gorfman. New York: Praeger, 91-101.

Taagepera, Rein y Matthew Shugart. 1989. Seats and Votes: The Effects and Determinants of Electoral Systems. New Heaven: Yale University Press.

Tsebelis, George. 2002. Veto Players. How Political Institutions Work. Princeton, NJ: Princeton University Press.

Weber, Max. 1984. Economía y sociedad. México: Fondo de Cultura Económica.

André Marenco es profesor del Programa de Posgrado en Ciencia Política de la Universidade Federal do Rio Grande do Sul (UFRGS), Brasil, y miembro del directorio de la Asociación Brasileña de Ciencia Política (2004-2008). Posee trabajos sobre reclutamiento, carreras legislativas, instituciones políticas y reforma electoral.

(E-mail: amarenco@terra.com.br) 
